OPEN ACCESS

Edited by:

Lauri A. Jensen-Campbell,

University of Texas at Arlington,

United States

Reviewed by:

William Tov,

Singapore Management University,

Singapore

Dominik Mischkowski,

Ohio University, United States

*Correspondence:

Joel O. Goldberg

jgoldber@yorku.ca

Specialty section:

This article was submitted to Personality and Social Psychology,

a section of the journal

Frontiers in Psychology

Received: 18 May 2020 Accepted: 23 November 2020 Published: 17 December 2020

Citation:

McComb SE, Goldberg JO, Flett GL and Rose AL (2020) The Double Jeopardy of Feeling Lonely and Unimportant: State and Trait Loneliness and Feelings and Fears of

Not Mattering.

Front. Psychol. 11:563420

doi: 10.3389/fpsyg.2020.563420

\section{The Double Jeopardy of Feeling Lonely and Unimportant: State and Trait Loneliness and Feelings and Fears of Not Mattering}

\author{
Sarah E. McComb, Joel O. Goldberg*, Gordon L. Flett and Alison L. Rose \\ Department of Psychology, York University, Toronto, ON, Canada
}

There have been recent concerns about an "epidemic of loneliness" during the pandemic, given the pervasiveness of loneliness in the population and its harmful effects on health and well-being. Therefore, it is important to establish the correlates of loneliness. The purpose of the current study was to explore how loneliness relates to a construct termed mattering, which is the feeling of being important to other people. Mattering was assessed with multiple measures in the current study (e.g., mattering in general, fears of not mattering, and mattering to peers). A sample of 172 female psychology undergraduate students aged 18-25 years completed self-report measures of general mattering, mattering to peers, anti-mattering, fear of not mattering, and state and trait loneliness. As predicted, lower levels of both general mattering and mattering to peers were associated with higher state loneliness. Higher feelings of anti-mattering (feelings of being invisible and insignificant to others) and fears of not mattering were associated with greater trait loneliness, as well as a reduced sense of mattering to friends. The findings illustrate that feeling as though one does not matter to others (i.e., feeling insignificant and unimportant) is associated with increased state and trait loneliness among young women. Implications are discussed for loneliness theory and how these results can enhance both clinical understanding and practice.

Keywords: loneliness, mattering, anti-mattering, friendships and peer relationships, peer isolation

\section{INTRODUCTION}

Loneliness is the distressing feeling that arises when one's desired quantity or quality of social connection has failed to be met (Peplau and Perlman, 1982). In recent years, concerns have been expressed by public health officials and researchers about an "epidemic of loneliness" given the pervasiveness of loneliness across the population and its debilitating effects (HoltLunstad, 2017; King, 2018). A recent study conducted in Canada found that $48 \%$ of Canadian adults reported feeling lonely, and $62 \%$ said they wished their friends and family would spend more time with them (Angus Reid Institute, 2019). Feelings of loneliness are present across the lifespan from adolescence through to old age, and while loneliness is often thought to be a condition that primarily afflicts the elderly, research polling has actually found that young women under the age of 25 report being the loneliest demographic group (Angus Reid Institute, 2019) 
and are thought to show particular susceptibility (Rokach, 2000). Despite young women being at high risk for experiencing loneliness very little research has been specifically conducted on experiences of loneliness and correlates of loneliness among young women, which is a gap the current study intends to address. Research on correlates of loneliness represents an important area of research given the negative consequences of loneliness, such as increased risk of depression, anxiety, poor immune functioning, and physical health, as well as earlier mortality (Uchino, 2006; Lasgaard et al., 2011; Cacioppo and Cacioppo, 2014; Hostinar et al., 2014; Rokach, 2019). Indeed, a recent study of patients receiving community mental health services found that loneliness predicted subsequent levels of four mental health indicators and was a better predictor than objective social isolation and social capital (see Wang et al., 2020).

Previous research has established that several interpersonal variables are associated risk factors for trait loneliness. Lack of social connection, such as living alone, being unmarried, not participating in social groups, and having fewer friends to turn to in times of need have all been associated with greater feelings of loneliness (Holt-Lunstad et al., 2010). Additionally, lack of social support from family, and especially from friends, was correlated with greater feelings of loneliness among young adults (Lee and Goldstein, 2016; Chang et al., 2017). Further, poor attachments to mothers and fathers, and especially peers, were all related to greater loneliness, as was low feelings of general belongingness (Yildiz, 2016).

Taken together, past research seems to reflect that feelings of belonging and social information from others regarding levels of interpersonal support, connection, and one's relative worth have an influence on trait loneliness. It stands to reason that feelings of mattering to others then may also be a relevant interpersonal predictor of loneliness. Mattering can be defined as the sense that other people find us important, depend on us, are interested in us, and care about what happens to us (Rosenberg and McCullough, 1981). If a person feels unimportant to others and as though others are not interested in them or care about what happens to them (i.e., he/she feels as though he/she does not matter to others), it follows that they may then feel a sense of loneliness.

Below we discuss mattering and loneliness from a historical perspective and a contemporary perspective. First, however, we describe the mattering construct in more detail. Rosenberg and McCullough (1981) introduced a form of relational mattering through their focus on the feeling and the need to be important to other people. The person who feels like she or he matters is someone who feels seen and heard by others who are valuing them. It is related to but more specific than Leary's notion of the sociometer, which blends being valued with others with openly being rejected or accepted by others and how this can impact self-esteem (see Leary, 2012; Leary and Acosta, 2018).

Key nuances help distinguish mattering from other constructs. For instance, Prilleltensky (2020) introduced an expanded view that sees mattering as being someone who is both valued by other people and who gives value to other people. This last component is important because it makes mattering less reactive and helps distinguish it from other constructs because people can become "mattering agents" who promote feelings of mattering among people in their social circle (for a related discussion, see Flett, 2018). Most notably, mattering is distinguishable from related constructs. It has been shown empirically in several studies that mattering is related to but distinct from self-esteem (e.g., Rosenberg and McCullough, 1981; Elliott et al., 2004; Dixon and Robinson Karpius, 2008; Flett et al., 2016b). A recent investigation by Flett and Nepon (2020) found that mattering and self-esteem were associated positively but mattering predicted significant variance in depression beyond self-esteem. Indeed, in their original paper, Rosenberg and McCullough (1981) showed in four samples of adolescents that after controlling for self-esteem, mattering was still a predictor of reduced levels of depression. There is also extensive evidence to support the conceptual and empirical distinction between mattering and related concepts such as belongingness and social support (see Elliott et al., 2004; Elliott, 2009; Flett, 2018). The distinction between the need to matter vs. the need to belong is perhaps best reflected by the person who feels part of a broader group and thus belongs but is not valued or extensively noticed within the group and thus feels a sense of not mattering to others.

\section{Mattering and Loneliness}

At present, to our knowledge, only one study thus far has examined the association between mattering and loneliness. Flett et al. (2016a) examined loneliness and mattering as part of a broader study. A sample of 232 undergraduate students completed various measures that included a measure of trait loneliness and the five-item General Mattering Scale (Marcus and Rosenberg, 1987). A robust negative association was found between mattering and loneliness $(r=-0.65)$, thus confirming that low feelings of general mattering to others was associated with greater trait loneliness among both men and women. Additional results indicated that feelings of not mattering partially mediated the link between a reported history of maltreatment and loneliness.

The current article re-examines and extends this association between feelings of not mattering and loneliness with multiple measures of each construct. While there has not been an extensive theoretical emphasis thus far on loneliness and mattering seen through a conceptual lens, some useful insights have been provided by Erich Fromm (1941). Flett (2018) describes in his broad review on the psychology of mattering, the influence that Fromm had on the mattering field and how his insights deserve more emphasis not only due to their influence on Rosenberg and McCullough (1981). Most notably, for our purposes, Fromm (1941) proposed that feelings of powerlessness (written presciently in the context of world events at the time) and personal insignificance (i.e., feelings of not mattering) are closely intertwined with feelings of loneliness. Indeed, the mandated exposure of individuals to social isolation around the globe due to the corona virusrelated public health crisis has reignited the need to explore correlates of loneliness and populations at specific risk (Flett and Zangeneh, 2020). Specifically, Flett and Zangeneh (2020) describe the vulnerability to psychological pain of those people who are alone and who feel unimportant, that their lives 
lack significance. Given these historical and contemporary observations, there is a clear need for programmatic research on loneliness and feelings of not mattering.

The current research was based on an expanded view of loneliness (i.e., state and trait loneliness) and mattering. We went beyond mattering in general to also assess mattering with respect to a specific relationship. To our knowledge, this topic has not been examined in terms of how feelings of mattering to specific others (e.g., mother, father, and peers) is related to loneliness. Given past research that has documented stronger associations between loneliness and lack of peer support or attachment, it is anticipated that low feelings of mattering to friends may be an especially important predictor of loneliness among young women and go beyond studies of basic belongingness in this population (Asher and Weeks, 2014).

We also examined mattering with additional focus on two recent extensions of the construct. The concept of anti-mattering is described in Flett (2018) and is evaluated using items that emphasize not mattering and feeling marginalized. According to Flett (2018), feelings of anti-mattering, are distinct from, and not merely the opposite of mattering, in keeping with the notion that constructs such as optimism and pessimism and hope vs. hopelessness are not mere endpoints of the same continuum. A recent review has summarized extensive evidence attesting to the incremental validity of this new measure of anti-mattering when considered along with the General Mattering Scale (see Flett and Nepon, 2020). Accordingly, we include a new measure of anti-mattering to evaluate whether it is also a relevant interpersonal correlate of loneliness. Anti-mattering can be defined as feeling insignificant and invisible to others and feeling as though no one cares about what you have to say or think. Those who strongly endorse feelings of antimattering may feel as though they do not matter at all to anyone. It stands to reason that those who feel as though they do not matter to anyone would be more likely to be dissatisfied with their social relationships and to feel lonely.

Further, we included a new measure of fear of not mattering to others that reflects the anxiety that people have about the possibility that they will not matter to others. This concept has been described by Casale and Flett (2020) as distinguishable from other interpersonally-based fears such as a fear of missing out or separation fears. The fear of not mattering is similar to other interpersonally-based constructs (e.g., rejection sensitivity, reassurance seeking, and fear of negative evaluation) in that it reflects unmet interpersonal needs and a need for validation through connection from others, but it is a specific fear reflecting a concern about not being valued and not being seen or heard by other people who show little interest. This emphasis on a fear of being or becoming insignificant to others is in keeping with research that ties individual differences in feelings of not mattering to others with anxious forms of insecure attachment. More generally, the fear of not mattering reflects the overlap that exists between the mattering and anxiety constructs and the anxious arousal and evaluation apprehension that accompanies a sense of not mattering (see Flett, 2019). Fear of not mattering to others may reflect a ruminative preoccupation about the threat of the depreciation of one's worth or value to others and the loss of important social relationships or resources. This fear of not mattering might be experienced by people who have lost people in their lives who were key sources of feelings of mattering. This type of fear could be relevant for older adults given the suggestion from Rosenberg and McCullough (1981) that feelings of mattering may be particularly relevant in understanding elderly people. Given these observations, fear of not mattering to others may be another correlate of loneliness. Certainly past research has found that rumination is associated with greater loneliness among young adults (Gan et al., 2015; Borawski, 2019).

\section{The Current Study}

In summary, the current study aimed to expand the sparse literature on how mattering is related to feelings of state and trait loneliness using a sample of young women aged 18-25 years old since they are considered to be the loneliest demographic group. The purpose of the current study was to examine how feelings of general mattering, mattering to peers, anti-mattering, and fear of not mattering related to reports of trait and state loneliness. Based on past research, the following hypotheses were tested: (1) Feelings of both general mattering and mattering to peers will be negatively associated with state and trait loneliness and (2) Feelings of anti-mattering and fear of not mattering will both be positively associated with state and trait loneliness. These hypotheses were based on past research. Our emphasis on going beyond trait loneliness to also consider state loneliness reflected our interest in assessing very current feelings of loneliness that could perhaps more closely reflect the current experience of young women as they adapt to the pandemic and its various challenges. It also reflects past research attesting to the usefulness of examining state loneliness as a form of reactivity in specific contexts (see van Roekel et al., 2018).

\section{MATERIALS AND METHODS}

\section{Participants}

Participants were 172 female psychology undergraduate students recruited through an online research participant pool at York University in Toronto, Canada. Inclusion criteria included being a biological female and being between the ages of 18-25 years. As previously mentioned, this sample was chosen because women in this age bracket have reported being the loneliest demographic group (Rokach, 2000; Angus Reid Institute, 2019). Participant ages ranged from $18-25$ years $(M=19.20, S D=1.68)$. The self-reported ethnic distribution of the sample was $34.7 \%$ Caucasian, 28.9\% South-Asian, 10.4\% East Asian, 9.8\% Middle Eastern, 5.8\% Black/African-Canadian, 1.2\% Pacific Islander, $0.6 \%$ Hispanic/Latino, and $8.1 \%$ identified as "Other." Most participants $(71.1 \%)$ reported completing a high school diploma as their highest level of completed education, followed by $19.7 \%$ who completed some college, $4.0 \%$ who completed a 2 -year degree, $4.0 \%$ who completed a 4 -year degree, and $0.6 \%$ who had completed a doctorate. 


\section{Measures}

\section{The General Mattering Scale}

The General Mattering Scale (GMS; Marcus and Rosenberg, 1987) is a brief five-item self-report scale that is used to measure how much one perceives that they matter to others at an overall level. Participants are asked to indicate how much they agree with each statement, such as "How important do you feel you are to other people?", by responding on a scale from $1=$ not at all to $4=a$ lot. Higher scores indicate greater perceived mattering. Internal consistency in the current study was good ( $\alpha=0.78)$. Extensive evidence attests to the reliability and validity of this measure (see Flett, 2018).

\section{The Mattering to Others Questionnaire}

The Mattering to Others Questionnaire (Marshall, 2001) is an 11-item self-report scale that can be used to assess perceived mattering to one's mother, father, or friends. We focused solely on assessing levels of mattering to friends/peers in the current study. Participants are asked to indicate how much they agree with each statement, such as "I matter to my friends," by responding on a scale from 1 = not much to $5=a$ lot. A mean score for all items are calculated and higher scores indicate greater perceived mattering. The level of internal consistency in the current study was somewhat low $(\alpha=0.59)$, which is uncharacteristic of the measure given that there is extensive psychometric support for this scale (see Flett, 2018).

\section{The Anti-Mattering Scale}

The Anti-Mattering Scale (AMS; Flett, 2018) is another brief five-item self-report scale that is used to measure anti-mattering at an overall level. The measure is designed to parallel the format of the GMS. Anti-mattering can be defined as a feeling of being insignificant and invisible to others. Participants are asked to "please choose the rating that you feel is the best for you," in regards to each item (e.g. "How much do you feel like you do not matter?"), by responding on a scale from $1=$ not at all to $4=a$ lot. Higher scores indicate greater perceived anti-mattering. Internal consistency in the current study was surprisingly low $(\alpha=0.58)$.

\section{The Fear of Not Mattering Inventory}

The Fear of Not Mattering Inventory (Besser et al., 2020) is a brief five-item self-report scale that is used to measure fear about not mattering to others. Participants are asked to indicate how much they agree with each statement, such as "To what extent are you afraid that you will not matter to other people?", by responding on a scale from $0=$ not at all to $3=$ almost all of the time. Higher scores indicate greater fear of not mattering. Internal consistency in the current study was excellent $(\alpha=0.91)$.

\section{UCLA Loneliness Scale}

The UCLA Loneliness Scale (Russell et al., 1980) is a 20-item self-report measure of trait loneliness. Participants are asked to indicate how often each of the statements listed is descriptive of themselves, ranging from $1=$ never to $4=$ often.
Higher scores are indicative of greater trait loneliness. Internal consistency in the current study was excellent $(\alpha=0.95)$.

\section{State Loneliness}

To measure state loneliness a single item visual analog scale was used. The scale consisted of a horizontal line with a slider bar, and endpoints labeled as "not at all" and "very much." Participants were asked to slide the bar to the point on the line that best represents how they felt in that moment in regards to the following statement: "I feel lonely." Responses to the scale could range from 0 to 100 , with higher scores indicating greater loneliness.

\section{Procedure}

Eligible participants could view and sign up for the study online through an online experiment management system. Upon sign up, participants gave their informed consent online. Participants then completed demographic questions and selfreport measures of state and trait feelings of loneliness, general mattering, mattering to others, anti-mattering, fear of not mattering, as well as several other questionnaires not pertinent to the current study. Participants completed the measure of state loneliness prior to all other questionnaires, so that state loneliness scores were not influenced or primed by questions on the trait loneliness or mattering measures. Participants then received online debriefing and partial course credit for their participation. This study's research protocol was approved by a university's ethics review board and conforms to the standards of the Canadian Tri-Council research ethics guidelines.

\section{Data Analysis}

All statistical analyses were conducted using SPSS version 25. Two separate multiple regression analyses were conducted to determine how general mattering, mattering to friends, antimattering, and fear of not mattering were related to both state and trait loneliness. A priori power analysis was conducted to determine the sample size needed to provide sufficient power $(0.80)$ to detect a moderate effect size $(0.25)$ at a significance level of alpha 0.05 for a multiple regression $F$-test analysis, using $G^{\star}$ Power 9 (Faul et al., 2007). The power analysis indicated that the minimum sample size needed would be 53 participants, which we exceeded.

\section{RESULTS}

\section{Bivariate Correlations}

Table 1 presents the means and standard deviations, as well as the correlations among all study variables. The means obtained in terms of levels of general mattering and antimattering are comparable to those summarized elsewhere (see Flett, 2018). It can be seen that state and trait loneliness are correlated substantially but not to the extent that they are redundant. As for the measures assessing the mattering construct, the correlations in Table $\mathbf{1}$ indicates that there is a substantial emphasis on the importance of fear of not mattering. 
TABLE 1 | Intercorrelations between state and trait loneliness, anti-mattering, and mattering-related variables.

\begin{tabular}{|c|c|c|c|c|c|c|c|c|}
\hline Variable & 1 & 2 & 3 & 4 & 5 & 6 & $M$ & $S D$ \\
\hline 1. State loneliness & - & & & & & & 33.40 & 32.72 \\
\hline 2. Trait loneliness & $0.63^{* *}$ & - & & & & & 21.42 & 14.42 \\
\hline 3. Mattering to friends & $-0.38^{* *}$ & $-0.70^{* *}$ & - & & & & 38.32 & 11.25 \\
\hline 4. General mattering & $-0.37^{* *}$ & $-0.50^{* * *}$ & $0.61^{* * *}$ & - & & & 14.21 & 2.95 \\
\hline 5. Anti-mattering & $0.26^{* *}$ & $0.42^{* *}$ & $-0.22^{* *}$ & -0.14 & - & & 12.99 & 2.86 \\
\hline 6. Fear of not mattering & $0.37^{* *}$ & $0.60^{* * *}$ & $-0.46^{* *}$ & $-0.44^{* *}$ & $0.44^{* *}$ & - & 6.40 & 3.84 \\
\hline
\end{tabular}

$N=172$. Range of possible scores for each construct are as follows: state loneliness: 0-100; trait loneliness: 0-63; mattering to friends; 11-55; general mattering: 5-20; Antimattering: 5-20; Fear of not mattering: $0-15 . " p<0.05 ;{ }^{* *} p<0.01$.

TABLE 2 | Multiple regression analyses predicting state and trait loneliness.

\begin{tabular}{lrccccc}
\hline & B & SE & $\boldsymbol{\beta}$ & $\boldsymbol{t}$ & $\boldsymbol{p}$ & $\mathbf{9 5 \%} \mathbf{~ C l}$ \\
\hline State loneliness & & & & & & \\
\hline General mattering & -1.98 & 0.99 & -0.18 & -2.01 & 0.04 & {$[-3.93,-0.04]$} \\
Mattering to friends & -0.53 & 0.26 & -0.18 & -2.01 & 0.04 & {$[-1.04,-0.01]$} \\
Anti-mattering & 1.59 & 0.87 & 0.14 & 1.82 & 0.07 & {$[-0.14,3.32]$} \\
Fear of not mattering & 1.14 & 0.74 & 0.13 & 1.54 & 0.12 & {$[-0.32,2.59]$} \\
$\quad$ Trait loneliness & & & & & & \\
General mattering & -0.26 & 0.30 & -0.05 & -0.86 & 0.39 & {$[-0.86,0.34]$} \\
Mattering to friends & -0.64 & 0.08 & -0.50 & -8.02 & 0.00 & {$[-0.80,-0.49]$} \\
Anti-mattering & 0.93 & 0.27 & 0.19 & 3.46 & 0.00 & {$[0.40,1.46]$} \\
Fear of not mattering & 1.00 & 0.23 & 0.27 & 4.43 & 0.00 & {$[0.56,1.45]$} \\
\hline
\end{tabular}

Specifically, the fear of not mattering with others showed significant negative associations with both general mattering $(r=-0.44)$ and mattering to friends $(r=-0.46)$ and it was associated positively to a comparable degree with anti-mattering $(r=0.44)$.

Regarding the associations between the indices of mattering and loneliness, it can be seen in Table 1 that state loneliness was negatively related to both general mattering and mattering to friends, with correlations of moderate effect sizes. Trait loneliness was also negatively related to both general mattering and mattering to friends, with correlations of large effect sizes, especially for mattering to friends. Both state and trait loneliness were positively related to anti-mattering and fear of not mattering, with stronger correlations for trait than state loneliness.

\section{Regression Analyses}

Table 2 presents the results of a multiple regression in which general mattering, mattering to friends, anti-mattering, and fear of not mattering are regressed onto state loneliness and trait loneliness in separate analyses. The analysis predicting state loneliness was significant, $F(4,167)=11.66, p<0.001$, and accounted for $22 \%$ of the variance. As seen in Table 2, and as expected, both general mattering and mattering to friends were significant negative predictors of state loneliness. However, anti-mattering and fear of not mattering were not significant predictors of state loneliness.

The same analysis was conducted to predict levels of trait loneliness. The analysis predicting trait loneliness was significant, $F(4,167)=67.44, p<0.001$, and accounted for $62 \%$ of the variance. It is important to underscore here that in this sample, the mattering variables were substantially more relevant to trait loneliness than state loneliness. As seen in Table 2, mattering to friends was a significant and negative predictor of trait loneliness, but contrary to predictions feelings of general mattering was not a significant predictor. Further, as predicted, both anti-mattering and fear of not mattering were significant and positive predictors of trait loneliness. Mattering to friends proved to be the most robust predictor of trait loneliness.

\section{DISCUSSION}

The purpose of the current study was to extend what is known thus far about the link between feelings of not mattering and loneliness. Our investigation focused on young women aged 18-25 years old, since recent research suggests they are the loneliest demographic group. Specifically, our aim was to examine how feelings of general mattering, mattering to peers, antimattering, and fear of not mattering related to young women's self-reported state and trait loneliness. This was the first study to examine how mattering, anti-mattering, and fear of not mattering was related to both state and trait loneliness. This focus on multiple elements of mattering and both trait and state loneliness reflected our goal of conducting the most extensive study thus far on mattering and loneliness.

Overall, the pattern of results indicated that variables tapping mattering were related broadly to loneliness, as was expected, though they were much more relevant to understanding trait loneliness. To some extent, this could reflect in part the methodological constraints of very different self-report formats used to assess trait loneliness vs. state loneliness in the current study. Also, as we discuss in more detail below, there was also evidence attesting to the merits of examining various components of the mattering construct in terms of how they relate to loneliness.

Several key findings emerged in terms of how mattering is related to state and trait loneliness. First, as predicted, we found that low feelings of general mattering and mattering to peers were associated with greater feelings of state loneliness among young women. However, in regard to trait loneliness, only low mattering to peers, and not general mattering, was a significant predictor of greater trait loneliness. Our findings from the regression analysis qualified and extended the finding of Flett et al. (2016a) who found that low feelings of general mattering were associated with greater trait loneliness among young women. While both general mattering and mattering 
to peers was a significant predictor of state loneliness, we found that only mattering to peers was a significant predictor of trait loneliness among young women. These results highlight the importance of feeling valued and important to peers, on protecting young women from feelings of loneliness. This finding is in line with past research, which has found that social support and positive relationships with peers is a protective factor against feelings of loneliness among young women (Lee and Goldstein, 2016; Yildiz, 2016; Chang et al., 2017), and that greater perceived mattering to peers is associated with better well-being (Matera et al., 2020). Feelings of mattering to peers may be especially important to women who are emerging adults, as this is a time in their life when many young women are moving away from home to live independently to pursue work or start post-secondary education. Therefore, they may be losing immediate contact with family, and relying on support from friends during this time (Lee and Goldstein, 2016).

The second key finding was that greater levels of antimattering were associated with greater trait loneliness (but not state loneliness). Therefore, unsurprisingly, results indicated that feeling as if one does not matter at all to anyone, and feeling insignificant and invisible to others, is associated with greater trait feelings of loneliness. As such, these findings extend past complementary research, which has found that low social support and connection are associated with greater loneliness among young adults (Holt-Lunstad et al., 2010; Lee and Goldstein, 2016). The current findings add to this body of research, by noting that it is not just low social connection that is linked with increased feelings of loneliness, but also perceived subjective feelings of being insignificant to those with whom one does have social contact.

Finally, greater fear of not mattering was associated with greater trait loneliness (but not state loneliness). Fears over not mattering may reflect a ruminative cognitive style about the negative consequences of not mattering to others, or coming to matter less than one currently does. Chronic engagement in this kind of rumination may result in increased feelings of trait loneliness over time, as one consistently reflects on how awful it would feel to not matter to others. In turn, it is possible that those who are already lonely are predisposed to ruminating about these kinds of concerns. This finding is consistent with past research that has found that increased rumination is associated with greater loneliness (Gan et al., 2015; Borawski, 2019).

Taken together, the findings provide evidence for theoretical formulations of loneliness. Loneliness is not merely about being physically isolated, but rather the condition of feeling alone - feeling disconnected from others, and feeling that no one cares (Rokach, 2019), as well as feeling unimportant. In addition, the results further our clinical understandings of loneliness in the high risk group of young women by pointing to a need to focus on their concerns about whether they matter to their peers as well as their fears of not mattering. The problem of loneliness has been previously identified as a public health concern (Leigh-Hunt et al., 2017) but one of the ironies of the current public health pandemic crisis is that it has now heightened awareness to mobilize community efforts to reach out in novel ways to our most isolated and loneliest individuals (Flett and Zangeneh, 2020). With respect to the heightened risk of young women, this may involve not only "connecting" using social media, but finding ways to deepen these connections, not just through the sheer number of "friends" accumulated on social media (Hood et al., 2018) but through meaningful listening and discovering ways of reaching out that show kindness, compassion and true caring for others.

There are several implications that follow from the current evidence of the extensive and robust links between loneliness and feelings and fears of not mattering. At a theoretical level, conceptual models of phenomena such as the link between loneliness and physical health problems should consider how loneliness and feelings of being unimportant may combine to produce outcomes from a mediator or moderator perspective. Similarly, at a practical level, interventions designed to enhance the mental health and degree of interpersonal relatedness of profoundly lonely people should perhaps consider whether loneliness is accompanied by a sense of having little perceived value to other people. Both themes need to be addressed in clinical and counseling interventions. Of course, longitudinal or experimental research on the relationship between mattering and loneliness would better help to inform these interventions, as the current data only examined cross-sectionally how mattering is related to loneliness and cannot discuss whether this relationship is causal or not.

Like all research, the findings of the current study must be taken into consideration with some limitations. First, the data collected were based on self-report responses, which are always prone to subjective biases. Second, it was a limitation that the mattering to friends and anti-mattering measures had somewhat low reliabilities in the current study, which could have reduced correlations and the relation between anti-mattering on state loneliness. Additionally, the study design was crosssectional and therefore the directionality of the relationships examined cannot be determined. While we proposed that low mattering, high anti-mattering, and fear of not mattering to others are correlates of loneliness that could increase risk for loneliness, it is also possible that loneliness could be a risk factor for decreased feelings of mattering, as well as increased anti-mattering and fear of not mattering. Longitudinal research is therefore needed to establish the directionality of the relationships examined, or experimental research to determine causality. While it was a strength of the study that we measured both state and trait loneliness and differentiated between two types of mattering, we only measured loneliness and mattering at one timepoint. Indeed, state loneliness was measured at a time prior to mandated physical isolation due to a public health crisis and most likely the ratings would have been quite different compared to a period of physical quarantine. Longitudinal research should be conducted that examines if mattering can predict loneliness over time, or vice versa. Also, it was the strength of the study that we investigated correlates for loneliness among young women, which is an under-researched demographic group in the loneliness literature. However, while our study only examined relations between loneliness and 
mattering in young women, we are confident that the findings of the current study would replicate among other demographic groups such as adolescents or the elderly, who also experience significant feelings of loneliness (Rokach, 2000; Fazio, 2009). In particular, feelings of mattering and fears of not mattering may be an especially important construct to study among the elderly, who often lose important social contacts as they age and experience greater loneliness. Past research has documented that loneliness increases from middle adulthood to old age, and that the loss of social contact through widowhood, having no spouse or cohabiting partner, and little contact with friends was associated with greater loneliness among the elderly (von Soest et al., 2020). Further, qualitative research among the elderly has documented that many elderly persons report gloomy feelings about not mattering and aching loneliness, which attributed to no longer having children who were dependent upon them and to losing their occupations through retirement (van Wijngaarden et al., 2015). Therefore, fears of not mattering may also be an especially relevant construct to study among those who are approaching old age and facing retirement. Feelings of mattering and fears of not mattering would also be relevant constructs to examine among adolescents who also report increased feelings of loneliness from childhood (Laursen and Hartl, 2013). Adolescence is a time where teens are trying to establish an identity separate from parents and where peer relations become increasingly important to selfdevelopment. Therefore, mattering to peers may be especially important in protecting against feelings of loneliness in adolescence (Laursen and Hartl, 2013).

In summary, the results of the current study confirmed that a reduced sense of mattering, especially to peers, and an increased sense of anti-mattering or fear of not mattering to others is associated with greater trait loneliness. Additional results showed that a reduced sense of general mattering and mattering to peers was associated with greater state loneliness. Overall, our results suggest that having a reduced sense of

\section{REFERENCES}

Angus Reid Institute, (2019). A portrait of social isolation and loneliness in Canada today. Available at: http://angusreid.org/social-isolation-loneliness-canada/ (Accessed April 23, 2019).

Asher, S. R., and Weeks, M. S. (2014). "Loneliness and belongingness in the college years" in The handbook of solitude. eds. R. J. Coplan and J. C. Bowker (New York: Wiley \& Sons), 283-301.

Besser, A., Flett, G. L., Nepon, T., and Zeigler-Hill, V. (2020). Personality, cognition, and adaptability to the COVID-19 pandemic: associations, with loneliness, distress, and positive and negative mood states. Int. J. Ment. Health Addiction. doi: 10.1007/s11469-020-00421-x

Borawski, B. (2019). Authenticity and rumination mediate the relationship between loneliness and well-being. Curr. Psychol., 1-10. doi: 10.1007/ s12144-019-00412-9

Cacioppo, J. T., and Cacioppo, S. (2014). Older adults reporting social isolation or loneliness show poorer cognitive function 4 years later. Evid. Based Nurs. 17, 59-60. doi: 10.1136/eb-2013-101379

Casale, S., and Flett, G. L. (2020). Interpersonally-based fears during the COVID-19 pandemic: reflections on the fear of missing out and the fear of not mattering constructs. Clin. Neuropsychiatry 17, 88-93. doi: 10.36131/CN20200211

Chang, E. C., Chang, O. D., Martos, T., Sallay, V., Lee, J., Stam, K., et al. (2017). Family support as a moderator of the relationship between loneliness being significant and cared for by others could lead to state and trait feelings of loneliness among young women. These findings highlight the importance of young women forming strong social relationships, particularly with peers who make them feel important and cared for, in order to protect themselves against feelings of loneliness. The current findings suggest that bolstering feelings of mattering through therapy and other treatment avenues may help young women who are seeking to overcome feelings of state and trait loneliness.

\section{DATA AVAILABILITY STATEMENT}

The datasets presented in this article are not readily available because the requesting source must be affiliated with an academic institution. Requests to access the datasets should be directed to the corresponding author jgoldber@yorku.ca.

\section{ETHICS STATEMENT}

The current study was reviewed and approved by the Human Participants Review Committee at York University. The patients/ participants provided their written informed consent to participate in this study.

\section{AUTHOR CONTRIBUTIONS}

SM collected the data and wrote the first draft under the direct supervision of JG in fulfillment of her graduate student academic breadth requirements with further supervisory committee membership guidance by GF. JG and GF supervised the data analysis and edited drafts of the manuscript. AR provided further editorial and literature review support. All authors contributed to the article and approved the submitted version.

and suicide risk in college students: having a supportive family matters! Fam. J. 25, 257-263. doi: 10.1177/1066480717711102

Dixon, S. K., and Robinson Karpius, S. E. (2008). Depression and college stress among university undergraduates: do mattering and self-esteem make a difference? J. Coll. Stud. Dev. 49, 412-424. doi: 10.1353/csd.0.0024

Elliott, G. C. (2009). Family matters: The importance of mattering to family in adolescence. Chichester, West Sussex: Wiley-Blackwell.

Elliott, G. C., Kao, S., and Grant, A. (2004). Mattering: empirical validation of a social-psychological concept. Self Identity 3, 339-354. doi: $10.1080 / 13576500444000119$

Faul, F., Erdfelder, E., Lang, A. -G., and Buchner, A. (2007). G*Power 3: a flexible statistical power analysis program for the social, behavioral, and biomedical sciences. Behav. Res. Methods 39, 175-191. doi: 10.3758/BF03193146

Fazio, E. M. (2009). "Sense of mattering in late life" in Advances in the conceptualization of the stress process: Essays in honor of Leonard I. Pearlin. eds. W. R. Avison, C. S. Aneshenel, S. Schieman and B. Wheaton (New York: Springer), 149-174.

Flett, G. L. (2018). The psychology of mattering: Understanding the human need to be significant. Cambridge, MA: Academic Press/Elsevier.

Flett, G. L. (2019). "Mattering, anxiety, and fear of failure in children and adolescents" in Perspectives on early childhood psychology and education. Vol. 4. ed. D. E. McIntosh (Pace University Press), 277-293.

Flett, G. L., Goldstein, A. L., Pechenkov, I. G., Nepon, T., and Wekerle, C. (2016a). Antecedents, correlates, and consequences of feeling like you don't 
matter: associations with maltreatment, loneliness, social anxiety, and the five-factor model. Personal. Individ. Differ. 92, 52-56. doi: 10.1016/j. paid.2015.12.014

Flett, G. L., and Nepon, T. (2020). Mattering versus self-esteem in university students: associations with regulatory focus, social feedback, and psychological distress. J. Psychoeduc. Assess. 38, 663-674. doi: 10.1177/0734282919890786

Flett, G. L., Su, C., Ma, L., and Guo, L. (2016b). Mattering as a unique resilience factor in Chinese children: a comparative analysis of predictors of depression. Int. J. Child Adolesc. Resil. 4, 91-102.

Flett, G. L., and Zangeneh, M. (2020). Mattering as a vital support for people during the COVID-19 pandemic: the benefits of feeling and knowing that someone cares during times of crisis. J. Concurrent Disord. 2, 106-123.

Fromm, E. (1941). Escape from freedom. New York: Norton.

Gan, P., Xie, Y., Duan, W., Deng, Q., and Yu, X. (2015). Rumination and loneliness independently predict six-month later depression symptoms among Chinese Elderly in Nursing Homes. PLoS One 10:e0137176. doi: 10.1371/ journal.pone.0137176

Holt-Lunstad, J. (2017). The potential public health relevance of social isolation and loneliness: prevalence, epidemiology, and risk factors. Public Policy Aging Rep. 27, 127-130. doi: 10.1093/ppar/prx030

Holt-Lunstad, J., Smith, T. B., and Layton, J. B. (2010). Social relationships and mortality risk: a meta-analytic review. PLoS Med. 7:e1000316. doi: 10.1371/journal.pmed.1000316

Hood, M., Creed, P. A., and Bianca, J. (2018). Loneliness and online friendships in emerging adults. Personal. Individ. Differ. 133, 96-102. doi: 10.1016/j.paid.2017. 03.045

Hostinar, C. E., Sullivan, R. M., and Gunnar, M. R. (2014). Psychobiological mechanisms underlying the social buffering of the hypothalamic-pituitaryadrenocortical axis: a review of animal models and human studies across development. Psychol. Bull. 140, 256-282. doi: 10.1037/a0032671

King, M. (2018). Working to address the loneliness epidemic: perspective-taking, presence, and self-disclosure. Am. J. Health Promot. 32, 1315-1317. doi: $10.1177 / 0890117118776735 \mathrm{c}$

Lasgaard, M., Goossens, L., and Elklit, A. (2011). Loneliness, depressive symptomology, and suicide ideation in adolescence: cross-sectional and longitudinal analyses. J. Abnorm. Child Psychol. 39, 137-150. doi: 10.1007/s10802-010-9442-x

Laursen, B., and Hartl, A. C. (2013). Understanding loneliness during adolescence: developmental changes that increase the risk of perceived social isolation. J. Adolesc. 36, 1261-1268. doi: 10.1016/j.adolescence.2013.06.003

Leary, M. R. (2012). Sociometer theory. in Handbook of theories of social psychology. Vol. 2, eds. L. Van Lange, A. W. Kruglanski and E. T. Higgins (London, UK: Sage Publications), 141-159.

Leary, M. R., and Acosta, J. (2018). "Acceptance, rejection, and the quest for relational value" in Cambridge handbook of personal relationships. eds. D. Perlman and A. Vangelisti (Cambridge, UK: Cambridge University Press), 378-390.

Lee, C. S., and Goldstein, S. E. (2016). Loneliness, stress, and social support in young adulthood: does the source of support matter? J. Youth Adolesc. 45, 568-580. doi: 10.1007/s10964-015-0395-9

Leigh-Hunt, N., Bagguley, D., Bash, K., Turner, V., Turnbull, S., Valtorta, N., et al. (2017). An overview of systematic reviews on the public health consequences of social isolation and loneliness. Public Health 152, 157-171. doi: $10.1016 /$ j.puhe.2017.07.035
Marcus, F. M., and Rosenberg, M. (1987). Mattering: It's measurement and significance in everyday life. Paper presented at the 57th annual Eastern Sociological Society Meeting. Boston, Massachusetts.

Marshall, S. K. (2001). Do I matter? Construct validation of adolescents' perceived mattering to parents and friends. J. Adolesc. 24, 473-490. doi: 10.1006/ jado.2001.0384

Matera, C., Bosco, M., and Meringolo, P. (2020). Perceiving mattering to family and friends, self-esteem, and well-being. Psychol. Health Med. 25, 550-558. doi: 10.1080/13548506.2019.1626454

Peplau, P. A., and Perlman, D. (1982). Loneliness: A sourcebook of current theory, research, and therapy. New York: Wiley-Interscience.

Prilleltensky, I. (2020). Mattering at the intersection of psychology, philosophy, and politics. Am. J. Community Psychol. 65, 16-34. doi: 10.1002/ajcp.12368

van Roekel, E., Verhagen, M., Engels, R. C. M. E., Scholte, R. H. J., Cacioppo, S., and Cacioppo, J. T. (2018). Trait and state levels of loneliness in early and late adolescents: examining the differential reactivity hypothesis. J. Clin. Child Adolesc. Psychol. 47, 888-899. doi: 10.1080/15374416.2016.1146993

Rokach, A. (2000). Perceived causes of loneliness in adulthood. J. Soc. Behav. Pers. 15, 67-84.

Rokach, A. (2019). Loneliness and social connection. Visions J. 14, 5-7.

Rosenberg, M., and McCullough, C. B. (1981). Mattering: inferred significance and mental health among adolescents. Res. Community Ment. Health 2, 163-182.

Russell, D., Peplau, L. A., and Cutrona, C. E. (1980). The revised UCLA loneliness scale: con- current and discriminant validity evidence. J. Pers. Soc. Psychol. 39, 472-480. doi: 10.1037/0022-3514.39.3.472

Uchino, B. N. (2006). Social support and health: a review of physiological processes potentially underlying links to disease outcomes. J. Behav. Med. 29, 377-387. doi: 10.1007/s10865-006-9056-5

van Wijngaarden, E., Leget, C., and Goossensen, A. (2015). Ready to give up on life: the lived experience of elderly people who feel life is completed and no longer worth living. Soc. Sci. Med. 138, 257-264. doi: 10.1016/j.socscimed.2015.05.015 von Soest, T., Hansen, T., Luhmann, M., and Gerstorf, D. (2020). Development of loneliness in midlife and old age: its nature and correlates. J. Pers. Soc. Psychol. 118, 388-406. doi: 10.1037/pspp0000219

Wang, J., Lloyd-Evans, B., Marston, L., Ma, R., Mann, F., Solmi, F., et al. (2020). Epidemiology of loneliness in a cohort of UK mental health community crisis service users. Soc. Psychiatry Psychiatr. Epidemiol. 55, 811-822. doi: 10.1007/s00127-019-01734-6

Yildiz, M. A. (2016). Serial multiple mediation of general belongingness and life satisfaction in the relationship between attachment and loneliness in adolescents. Educ. Sci. Theory Pract. 16, 553-578. doi: 10.12738/estp.2016.2.0380

Conflict of Interest: The authors declare that the research was conducted in the absence of any commercial or financial relationships that could be construed as a potential conflict of interest.

Copyright (c) 2020 McComb, Goldberg, Flett and Rose. This is an open-access article distributed under the terms of the Creative Commons Attribution License (CC BY). The use, distribution or reproduction in other forums is permitted, provided the original author(s) and the copyright owner(s) are credited and that the original publication in this journal is cited, in accordance with accepted academic practice. No use, distribution or reproduction is permitted which does not comply with these terms. 